\title{
CRITICAL CASES IN SLOW/FAST CONTROL PROBLEMS
}

\author{
V.A. Sobolev \\ Samara National Research University, Samara, Russia
}

\begin{abstract}
The aim of the paper is to describe the main critical cases in the theory of singularly perturbed optimal control problems and to give examples which are typical for slow/fast systems. The theory has traditionally dealt only with perturbation problems near normally hyperbolic manifold of singularities and this manifold is supposed to isolated. We reduce the original singularly perturbed problem to a regularized one such that the existence of slow integral manifolds can be established by means of the standard theory. We illustrate our approach by several examples of control problems.
\end{abstract}

Keywords: integral manifolds, singular perturbations, optimal control

Citation: Sobolev VA. Critical cases in slow/fast control problems. CEUR Workshop Proceedings, 2016; 1638: 742-753. DOI: 10.18287/1613-0073-2016-1638-742-753

\section{Introduction}

Consider singularly perturbed differential systems of the type

$$
\frac{d x}{d t}=f(x, y, t, \varepsilon), \quad \varepsilon \frac{d y}{d t}=g(x, y, t, \varepsilon)
$$

with vector variables $x$ and $y$, and a small positive parameter $\varepsilon$.

Such systems play an important role as mathematical models of numerous nonlinear phenomena in different fields (see e.g. [1-7]).

A usual approach in the qualitative study of (1) is to consider first the so called degenerate system

$$
\frac{d x}{d t}=f(x, y, t, 0), \quad 0=g(x, y, t, 0)
$$

and then to draw conclusions for the qualitative behavior of the full system (1) for sufficiently small $\varepsilon$. In order to recall a basic result of the geometric theory of singularly perturbed systems we introduce the following notation and assumptions for sufficiently small positive $\varepsilon_{0}, 0 \leq \varepsilon \leq \varepsilon_{0}$.

$\left(A_{1}\right)$. Functions $f$ and $g$ are sufficiently smooth and uniformly bounded together with all their derivatives.

$\left(A_{2}\right)$. There are some region $\mathcal{G} \in R^{m}$ and a function $h(x, t, \varepsilon)$ of the same smoothness as $g$ such that

$$
g(x, h(x, t), t, 0) \equiv 0 \quad \forall(x, t) \in \mathcal{G} \times R .
$$


$\left(A_{3}\right)$. The spectrum of the Jacobian matrix $B(x, t)=g_{y}(x, h(x, t), t, 0)$ is uniformly separated from the imaginary axis for all $(x, t) \in \mathcal{G} \times R$, i.e. the eigenvalues $\lambda_{i}(x, t)(i=1, \ldots, n)$ of the matrix $B(x, t)$ satisfy the inequality

$$
\left|\operatorname{Re} \lambda_{i}(x, t)\right| \geq \gamma
$$

for some positive number $\gamma$.

Then the following result is valid (see e.g. $[8,9]$ ):

Proposition 1. Under the assumptions $\left(A_{1}\right)-\left(A_{3}\right)$ there is a sufficiently small positive $\varepsilon_{1}, \varepsilon_{1} \leq \varepsilon_{0}$, such that for $\varepsilon \in \overline{I_{1}}$ system (1) has a smooth integral manifold $\mathcal{M}_{\varepsilon}$ (slow integral manifold) with the representation

$$
\mathcal{M}_{\varepsilon}:=\left\{(x, y, t) \in R^{n+m+1}: y=\psi(x, t, \varepsilon),(x, t) \in \mathcal{G} \times R\right\}
$$

and with the asymptotic expansion

$$
\psi(x, t, \varepsilon)=h(x, t)+\varepsilon \psi_{1}(x, t)+\ldots .
$$

The motion on this manifold is described by the slow differential equation

$\dot{x}=f(x, \psi(x, t, \varepsilon), t, \varepsilon)$.

Remark 1. The global boundedness assumption in $\left(A_{1}\right)$ with respect to $(x, y)$ can be relaxed by modifying $f$ and $g$ outside some bounded region of $R^{n} \times R^{m}$.

Remark 2. In applications it is usually assumed that the spectrum of the Jacobian matrix $g_{y}(x, h(x, t), t, 0)$ is located in the left half plane. Under this additional hypothesis the manifold $\mathcal{M}_{\varepsilon}$ is exponentially attracting for $\varepsilon \in I_{1}$.

The case that assumption $\left(A_{3}\right)$ is violated is called critical. We distinguish three subcases:

1. The Jacobian matrix $g_{y}(x, y, t, 0)$ is singular on some subspace of $R^{m} \times$ $R^{n} \times R$. In that case, system (1) is referred to as a singular singularly perturbed system [10]. This subcase has been treated in $[3,4,7,10,11]$.

2. The Jacobian matrix $g_{y}(x, y, t, 0)$ has eigenvalues on the imaginary axis with nonvanishing imaginary parts. A similar case has been investigated in $[3,4,12]$.

3. The Jacobian matrix $g_{y}(x, y, t, 0)$ is singular on the set $\mathcal{M}_{0}:=$ $\left\{(x, y, t) \in R^{m} \times R^{n} \times R: y=h(x, t),(x, t) \in \mathcal{G} \times R\right\}$. In that case, $y=$ $h(x, t)$ is generically an isolated root of $g=0$ but not a simple one.

Other critical cases were considered, for example, in [3,4,13-21].

The critical case (i) is considered in Section 2 as applied to the high-gain control problem, the case (ii) is considered in Section 3 as applied to the manipulator control, the case (iii) is considered in Section 4 as applied to the partially cheap control problem. Two critical cases, (i) and (ii) are combined in the the optimal control problem which is analyzed in Section 5, and therefore it is possible to say that this Section is devoted to the consideration of the twice critical case. It is not inconceivable that combinations of other pairs of critical cases and even triple critical case are of interest as well and possibly they will be considered later. 


\section{Singular singularly perturbed systems}

For a better idea of the problems we wish to examine, and to gain some insight into why the term in the title is used, we initially consider the following differential system

$$
\varepsilon \dot{z}_{1}=2 z_{1}-z_{2}, \quad \varepsilon \dot{z}_{2}=(6+3 \varepsilon) z_{1}-3 z_{2},
$$

or, in the vector form

$$
\varepsilon\left(\begin{array}{c}
\dot{z}_{1} \\
\dot{z}_{2}
\end{array}\right)=A(\varepsilon)\left(\begin{array}{c}
z_{1} \\
z_{2}
\end{array}\right)
$$

where

$$
A(\varepsilon)=\left(\begin{array}{cc}
2 & -1 \\
6+3 \varepsilon & -3
\end{array}\right) .
$$

At first glance it would seem that there are two fast variables $z_{1}$ and $z_{2}$ and we apply the proposed approach to the analysis of this system. Setting $\varepsilon$ equal to zero we obtain the linear algebraic system

$$
2 z_{1}-z_{2}=0, \quad 6 z_{1}-3 z_{2}=0 .
$$

Apart from the trivial solution this system possesses an one-parameter family of solutions $z_{1}=s, z_{2}=2 s$, where $s$ is a real parameter. Thus there is no isolated solution to the degenerate system. The reason is that the matrix is singular, i.e. $\operatorname{det} A(0)=0$ and the singularly perturbed system in this case is called a singular singularly perturbed system. In fact, in this particular system it is possible to extract a slow variable and obtain a system with one slow and one fast variable. Taking into account that the rows of matrix $A$ are proportional for $\varepsilon=0$ (proportionality constant is equal 3 ), we introduce a new variable $x=z_{2}-3 z_{1}$, and obtain the following differential equation for the slow variable $\dot{x}=-x+z_{2}$. To obtain the full solution, it is possible to use either of the two equations for $z_{1}$ or $z_{2}$ as a fast equation. If we choose the equation for $z_{2}$, then, after taking into account $x-z_{2}=-3 z_{1}$, we obtain the singularly perturbed equation $\varepsilon \dot{z}_{2}=-(2+\varepsilon) x-(1-\varepsilon) z_{2}$. As a result we obtain the system

$$
\dot{x}=-x+z_{2}, \quad \varepsilon \dot{z}_{2}=-(2+\varepsilon) x-(1-\varepsilon) z_{2},
$$

which has the form (1). It is easy to check by direct substitution into the invariance equation that this last system possesses the one-dimensional attractive slow invariant manifold $z_{2}=k x$. On substituting for $z_{2}$ the above equations imply

$$
\varepsilon k(-1+k) x=-(2+\varepsilon) x-(1-\varepsilon) k x,
$$

and this implies

$$
\varepsilon k^{2}+(1-2 \varepsilon) k+2+\varepsilon=0 .
$$

Setting in the last equation $k=k_{0}+\varepsilon k_{1}+O\left(\varepsilon^{2}\right)$ and equating the powers of $\varepsilon$, we obtain $k_{0}=-2, k_{1}=-9$. Thus, the invariant manifold has the form

$$
z_{2}=-\left(2+9 \varepsilon+O\left(\varepsilon^{2}\right)\right) x=-\left(2+9 \varepsilon+O\left(\varepsilon^{2}\right)\right)\left(z_{2}-3 z_{1}\right),
$$

or, in equivalent form

$$
z_{2}=\left(2+3 \varepsilon+O\left(\varepsilon^{2}\right)\right) z_{1} .
$$




\section{High-gain control}

Consider the control system

$\dot{x}=\zeta(x)+B(x) u, x(0)=x_{0}$,

where $x \in R^{n}, u \in R^{r}$ and $t \geq 0$. The vector function $\zeta$ and the matrix function $B$ are taken to be sufficiently smooth and bounded. The control vector $u$ is to be selected in such a way as to transfer the vector $x$ from $x=x_{0}$ to a sufficiently small neighborhood of a smooth $m$-dimensional surface $S(x)=0$. A commonly employed feedback control is

$u=-\frac{1}{\varepsilon} K S(x)$,

where $K$ is a constant $r \times m-$ matrix and $\varepsilon$ is a small positive parameter, see [6] and references therein.

Suppose that we can choose the matrix $K$ in such a way that the matrix $-N(x, t)=-G B K$ is stable, i.e., all eigenvalues have strictly negative real parts, and its inverse matrix is bounded, and introduce the additional variable $y=S(x)$, then $x$ and $y$ satisfy the system

$$
\begin{aligned}
& \varepsilon \dot{x}=\varepsilon \zeta(x)-B(x) K y, \quad x(0)=x_{0}, \\
& \varepsilon \dot{y}=\varepsilon G(x) \zeta(x)-G(x) B(x) K y, \quad y(0)=y_{0}=S\left(x_{0}\right),
\end{aligned}
$$

where $G(x)=\partial S / \partial x$. The reduced $(\varepsilon=0)$ algebraic problem possesses an $n$-parameter family of solutions $x=v, \quad y=0$. The role of $A$ is played by the singular matrix

$$
\left(\begin{array}{cc}
0 & -B K \\
0 & -N
\end{array}\right) \text {. }
$$

The latter singular singularly perturbed differential system possesses an $n-$ dimensional slow integral manifold

$$
x=v, \quad y=\varepsilon N^{-1}(v, t) G(v) \zeta(v)+O\left(\varepsilon^{2}\right) .
$$

The flow on the manifold is governed by

$$
\dot{v}=\left[I-B(v) K N^{-1}(v) G(v)\right] \zeta(v)+O(\varepsilon) .
$$

Introduce the new variable

$$
y=z+\varepsilon N^{-1}(x) G(x) \zeta(x) .
$$

Then for $z$ we obtain the equations

$$
\varepsilon \dot{z}=-N(x) z+O(\varepsilon) .
$$

It is now clear that the representations

$$
y=N^{-1} G \zeta+O(\varepsilon)
$$

are valid for for all $t>0$. Thus, under the control law (5) the trajectory very quickly attains the $\varepsilon$-neighborhood of $S(x)=0$. 
Let us introduce the modified control

$$
u=-\frac{1}{\varepsilon} K\left[S(x)-\varepsilon N^{-1}(x) G(x) \zeta(x)\right],
$$

with the stable matrix $-N(x)=-G B K$. Under this control for the variable $x$ we obtain the equation

$$
\varepsilon \dot{x}=\varepsilon\left[I-B(x) K(G B K)^{-1} G(x)\right] \zeta(x)-B(x) K S(x),
$$

and for the variable $y=S(x)$ we obtain the equation

$$
\begin{aligned}
& \varepsilon \dot{y}=-N(x) y, \\
& y=O\left(e^{-\nu \varepsilon^{-1} t}\right), \quad \nu>0, t>0, \varepsilon \rightarrow 0
\end{aligned}
$$

for some positive $\nu$. Note that the modified control law turns $y \equiv 0$ invariant and exponentially attractive. This means that the modified control law (6) is more preferable than the usually used law (5). Surprisingly, there is no need for using of asymptotic expansion to design the control law since the modified control law gives the exact result.

\section{Weakly attractive integral manifolds}

In this section we consider the system (1) when the matrix $B=g_{y}(x, \phi(x, t), t, 0)$ has eigenvalues on the imaginary axis with nonvanishing imaginary parts. If the eigenvalues at $\varepsilon=0$ are pure imaginary but after taking into account the perturbations of higher order they move to the complex left half-plane, then the system under consideration has stable slow integral manifolds. It seems reasonable to say that this kind of problem for gyroscopic systems was previously investigated by the integral manifolds method, see, for example, [12]. Note that in the eigenvalues of the matrix of the linearized fast subsystem in such problems have the form $-\alpha \pm i \beta / \varepsilon$ with positive $\alpha$ and the existence problem for slow integral manifolds has not any connections with the bifurcation problems when the real parts of the eigenvalues change their sign. Some problems of the mechanics of manipulators with high-frequency and weakly damped transient regimes are now discussed in this context. More results along this line can be found in $[3,4,12]$.

\section{Control of a one rigid-link flexible-joint manipulator}

Consider a simple model of a rigid-link flexible joint manipulator [22,23], where $J_{m}$ is the motor inertia, $J_{1}$ is the link inertia, $M$ is the link mass, $l$ is the link length, $c$ is the damping coefficient, $k$ is the stiffness. The model is described by the equations:

$$
\begin{aligned}
& J_{1} \ddot{q}_{1}+M g l \sin q_{1}+c\left(\dot{q}_{1}-\dot{q}_{m}\right)+k\left(q_{1}-q_{m}\right)=0, \\
& J_{m} \ddot{q}_{m}-c\left(\dot{q}_{1}-\dot{q}_{m}\right)-k\left(q_{1}-q_{m}\right)=u .
\end{aligned}
$$

Here $q_{1}$ is the link angle, $q_{m}$ is the rotor angle, and $u$ is the torque input which is the controller.

The control problem under consideration consists of a tracking problem in which it is desired that the link coordinate $q_{1}$ follows a time-varying smooth and bounded desired trajectory $q_{d}(t)$ so that $\left|q_{d}(t)-q_{1}(t)\right| \rightarrow 0$ as $t \rightarrow \infty[22,23]$. 
If we rewrite the original system in the form

$$
\begin{aligned}
& J_{1} \ddot{q}_{1}+J_{m} \ddot{q}_{m}+M g l \sin q_{1}=u, \\
& \ddot{q}_{1}-\ddot{q}_{m}+\frac{M g l}{J_{1}} \sin q_{1}+k\left(\frac{1}{J_{1}}+\frac{1}{J_{m}}\right)\left(q_{1}-q_{m}\right) \\
& +c\left(\frac{1}{J_{1}}+\frac{1}{J_{m}}\right)\left(\dot{q}_{1}-\dot{q}_{m}\right)=-\frac{u}{J_{m}},
\end{aligned}
$$

then the use of the small parameter $\varepsilon=1 / \sqrt{k}$ and new variables

$$
x_{1}=\left(J_{1} q_{1}+J_{m} q_{m}\right) /\left(J_{1}+J_{m}\right), \quad x_{2}=\dot{x}_{1}, \quad y_{1}=q_{1}-q_{m}, \quad y_{2}=\varepsilon \dot{y}_{1},
$$

yield the system

$$
\begin{aligned}
& \dot{x}_{1}=x_{2}, \quad \dot{x}_{2}=-\frac{M g l}{J_{1}+J_{m}} \sin \left(x_{1}+\frac{J_{m}}{J_{1}+J_{m}} y_{1}\right)+\frac{u}{J_{1}+J_{m}}, \\
& \varepsilon \dot{y}_{1}=y_{2}, \quad \varepsilon \dot{y}_{2}=-\left(\frac{1}{J_{1}}+\frac{1}{J_{m}}\right) y_{1}-\varepsilon c\left(\frac{1}{J_{1}}+\frac{1}{J_{m}}\right) y_{2}- \\
& \varepsilon^{2} \frac{M g l}{J_{1}} \sin \left(x_{1}+\frac{J_{m}}{J_{1}+J_{m}} y_{1}\right)-\varepsilon^{2} \frac{u}{J_{m}} .
\end{aligned}
$$

Note that neglecting all terms of order $O\left(\varepsilon^{2}\right)$ in the r.h.s. of the last equation we obtain the independent subsystem

$$
\begin{aligned}
& \dot{\varepsilon} y_{1}=y_{2}, \\
& \varepsilon \dot{y}_{2}=-\left(\frac{1}{J_{1}}+\frac{1}{J_{m}}\right) y_{1}-\varepsilon c\left(\frac{1}{J_{1}}+\frac{1}{J_{m}}\right) y_{2},
\end{aligned}
$$

solutions of which are characterized by high frequency $\approx \sqrt{\left(1 / J_{1}+1 / J_{m}\right)} / \varepsilon$ and relatively slow decay $c\left(1 / J_{1}+1 / J_{m}\right) / 2$, since this differential system has the characteristic polynomial

$$
\varepsilon^{2} \lambda^{2}+c\left(\frac{1}{J_{1}}+\frac{1}{J_{m}}\right) \lambda+\left(\frac{1}{J_{1}}+\frac{1}{J_{m}}\right)
$$

which possesses complex zeros

$$
\lambda_{1,2}=-\frac{c}{2}\left(\frac{1}{J_{1}}+\frac{1}{J_{m}}\right) \pm \frac{i}{\varepsilon} \sqrt{\left(\frac{1}{J_{1}}+\frac{1}{J_{m}}\right)-\varepsilon^{2} \frac{c^{2}}{4}\left(\frac{1}{J_{1}}+\frac{1}{J_{m}}\right)^{2}} .
$$

Since the real part of these numbers is negative, for the analysis of the manipulator model under consideration it is possible to use the slow invariant manifold noting that the reducibility principle holds for this manifold (the exact statement may be found in [3]). The terms of $O\left(\varepsilon^{2}\right)$ of the subsystem (9) lead us to conclude that the slow invariant manifold may be found in the form $y_{1}=\varepsilon^{2} Y+O\left(\varepsilon^{3}\right)$ and $y_{2}=O\left(\varepsilon^{3}\right)$, where

$$
Y=-\left[\frac{M g l}{J_{1}} \sin \left(x_{1}\right)+\frac{u_{0}}{J_{m}}\right]\left(\frac{1}{J_{1}}+\frac{1}{J_{m}}\right)^{-1} \text {. }
$$


Here we used the representation $u=u_{0}+\varepsilon^{2} u_{1}+O\left(\varepsilon^{3}\right)$. Thus, the flow on this manifold is described by equations

$$
\dot{x}_{1}=x_{2}, \quad \dot{x}_{2}=-\frac{M g l}{J_{1}+J_{m}} \sin \left(x_{1}+\varepsilon^{2} \frac{J_{m}}{J_{1}+J_{m}} Y\right)+\frac{u_{0}+\varepsilon^{2} u_{1}}{J_{1}+J_{m}}+O\left(\varepsilon^{3}\right) .
$$

It is important to emphasize that due to (7) $q_{1}=x_{1}+\frac{J_{m}}{J_{1}+J_{m}} y_{1}$, where $y_{1}=$ $\varepsilon^{2} Y+O\left(\varepsilon^{3}\right)$, and on the slow invariant manifold we obtain the representation

$$
q_{1}=x_{1}+\varepsilon^{2} \frac{J_{m}}{J_{1}+J_{m}} Y+O\left(\varepsilon^{3}\right) .
$$

This allows us to rewrite the system (11) on the slow invariant manifold using the original variable $q_{1}$ instead $x_{1}$ in the form

$$
\ddot{q}_{1}-\varepsilon^{2} \frac{J_{m}}{J_{1}+J_{m}} \ddot{Y}=-\frac{M g l}{J_{1}+J_{m}} \sin \left(q_{1}\right)+\frac{u_{0}+\varepsilon^{2} u_{1}}{J_{1}+J_{m}}+O\left(\varepsilon^{3}\right) .
$$

\section{The case of multiple root of the degenerate equation}

We consider system (1) under the assumptions $\left(A_{1}\right)$ and $\left(A_{2}\right)$. Instead of hypothesis $\left(A_{3}\right)$ we suppose

$\operatorname{det} g_{y}(x, h(x, t), t, 0) \equiv 0 \quad \forall(x, t) \in \mathcal{G} \times R$,

that is, $y=h(x, t)$ is not a simple root of the degenerate equation

$g(x, y, t, 0)=0$.

Under this assumption we cannot apply Proposition 1.1 to system (1) in order to establish the existence of a slow integral manifold near $\mathcal{M}_{0}$ for small $\varepsilon$. Our goal is to derive conditions which imply that for sufficiently small $\varepsilon$ system (1) has at least one integral manifold $\mathcal{M}_{\varepsilon}$ with the representation

$$
y=\psi_{i}(x, t, \varepsilon)=h(x, t)+\varepsilon^{q_{i}} h_{1, i}(x, t)+\varepsilon^{2 q_{i}} h_{2, i}(x, t)+\ldots .
$$

where $q_{i}, 0<q_{i}<1$, is a rational number.

The key idea to solve this problem consists in looking for scalings and transformations of the type

$$
\varepsilon=\mu^{r}, y=\tilde{y}(\mu, z, x, t), t=\tilde{t}(\mu, \tau)
$$

such that system (1) can be reduced to a system

$$
\frac{d x}{d \tau}=f(x, z, \tau, \mu), \quad \mu \frac{d z}{d \tau}=g(x, z, \tau, \mu)
$$

to which Proposition 1.1 can be applied.

\section{Thrice critical case}

Consider the control system

$$
\varepsilon \dot{x}=A(t, \varepsilon) x+\varepsilon B(t, \varepsilon) u, \quad x \in R^{n+m}, \quad x(0)=x_{0}
$$


with the cost functional

$$
J=\frac{1}{2} x^{T}(1) F x(1)+\frac{1}{2} \int_{0}^{1}\left(x^{T}(t) Q(t) x(t)+\varepsilon u^{T}(t) R(t) u(t)\right) d t .
$$

where $A, F_{1}, Q$ are $(n \times n)$-matrices, $B$ is $(n \times m)$-matrix, and $R$ is $(m \times m)$ matrix. Suppose that all these matrices have the following asymptotic presentations with respect to $\varepsilon$ :

$$
\begin{aligned}
& A(t, \varepsilon)=\sum_{j \geq 0} \varepsilon^{j} A j(t), \\
& B(t, \varepsilon)=\sum_{j \geq 0} \varepsilon^{j} B_{j}(t), \\
& Q(t, \varepsilon)=\sum_{j \geq 0} \varepsilon^{j} Q_{j}(t), \\
& R(t, \varepsilon)=\sum_{j \geq 0} \varepsilon^{j} R_{j}(t), \\
& F(\varepsilon)=\sum_{j \geq 0} \varepsilon^{j} F_{j}
\end{aligned}
$$

with smooth on $t$ matrix coefficients, $t \in[0,1]$.

The solution of this problem is the optimal linear feedback control law

$u=-\varepsilon^{-1} R^{-1} B^{T} P(t, \varepsilon) x$,

where $P$ satisfies the differential matrix Riccati equation

$$
\varepsilon \dot{P}=-P A-A^{T} P+P S P-\varepsilon Q, \quad P(1, \varepsilon)=F .
$$

Setting $\varepsilon=0$ we obtain the matrix algebraic equation

$-M A_{0}-A_{0}^{T} M+M S_{0} M=0$,

where $S_{0}=B_{0} R_{0}^{-1} B_{0}^{T}$. It is clear that the main role plays the linear operator

$\mathbf{L} X=X A_{0}+A_{0}^{T} X$.

For this class of systems the eigenvalues of $A_{0}$ are pure imaginary and the spectrum of the linear operator $\mathbf{L}$ has a nontrivial kernel, since sums $\left(\lambda_{i}(t)+\right.$ $\left.\lambda_{j}(t)\right), i, j=1, \ldots, n$, form its spectrum. This means that the Riccati equation is singular singularly perturbed. Thus, the problem under consideration is critical in this sense. Moreover, under taking into account that zero eigenvalues are multiple and all other, nonzero eigenvalues of $\mathbf{L}$, are pure imaginary, it is possible to say that this problem is thrice critical. 


\section{Example}

Let

$$
\begin{aligned}
& A=\left(\begin{array}{cc}
-\varepsilon & 1 \\
-1 & -\varepsilon
\end{array}\right), \quad B=\left(\begin{array}{l}
0 \\
1
\end{array}\right), \quad R=(1), \quad Q=\left(\begin{array}{ll}
1 & 0 \\
0 & 0
\end{array}\right), \\
& S=\left(\begin{array}{ll}
0 & 0 \\
0 & 1
\end{array}\right), \quad P=\left(\begin{array}{ll}
p_{1} & p_{2} \\
p_{2} & p_{3}
\end{array}\right) .
\end{aligned}
$$

Consider the corresponding differential system

$$
\begin{aligned}
& \varepsilon \dot{p}_{1}=2 p_{2}+2 \varepsilon p_{1}+p_{2}^{2}-\varepsilon, \\
& \varepsilon \dot{p}_{2}=2 \varepsilon p_{2}-p_{1}+p_{3}+p_{2} p_{3}, \\
& \varepsilon \dot{p}_{3}=-2 p_{2}+2 \varepsilon p_{3}+p_{3}^{2} .
\end{aligned}
$$

First, we need to separate it into a slow and a fast subsystem. At first glance, all three equations are singularly perturbed. However, setting $\varepsilon=0$ we obtain $p_{1}=p_{2}=p_{3}=0$, and we should consider the matrix of leading terms on the right hand side of the system, which has the form

$$
\left(\begin{array}{ccc}
0 & 2 & 0 \\
-1 & 0 & 1 \\
0 & -2 & 0
\end{array}\right) .
$$

Obviously, this matrix has a zero eigenvalue and two pure imaginary eigenvalues, i.e. the problem under consideration is twice critical.Moreover, the trivial solution is multiple. This means that we have thrice critical case.

Let $\varepsilon=\mu^{2}$. Introducing the new variables

$$
p_{1}=\mu^{2} q_{1}+\mu, \quad p_{2}=\mu^{2} q_{2}+\mu^{2} / 2, \quad p_{3}=\mu^{2} q_{3}+\mu,
$$

and then $s=q_{1}+q_{3}$, we obtain the differential system

$$
\begin{aligned}
& \mu \dot{s}=2 q_{3}+\mu q_{2}+2 \mu s+\mu q_{2}^{2}+\mu q_{3}^{2}+4+\mu / 4, \\
& \mu^{2} \dot{q}_{2}=-s+2 \mu^{2} q_{2}+2 q_{3}+\mu q_{2}+\mu^{2} q_{2} q_{3}+\mu / 2+\mu^{2}, \\
& \mu^{2} \dot{q}_{3}=-2 q_{2}+2 \mu q_{3}+2 \mu^{2} q_{3}+\mu^{2} q_{3}^{2}+2 \mu
\end{aligned}
$$

with the slow variable $s$ and two fast variables $q_{2}, q_{3}$.

The last system possesses one-dimensional slow invariant manifold which is weakly attractive with respect to argument $1-t$ because the main matrix of the fast subsystem is

$$
\left(\begin{array}{cc}
\mu & 2 \\
-2 & 2 \mu
\end{array}\right) \text {. }
$$

Thus, the dimension of the system of Riccati differential equations can be reduced from three to one. Let us construct the slow integral manifold using the fact that it can be asymptotically expanded in powers of the small parameter. Setting

$$
q_{2}=\varphi(s, \mu)=\mu \varphi_{1}(s)+\mu^{2} \ldots,
$$


$q_{3}=\psi(s, \mu)=\psi_{0}(s)+\mu \psi_{1}(s)+\mu^{2} \ldots$,

we obtain

$\psi_{0}(s)=s / 2, \quad \varphi_{1}(s)=s / 2, \quad \psi_{1}(s)=-1 / 4$.

Thus we obtain the slow invariant manifold

$q_{2}=\mu s / 2+O\left(\mu^{2}\right), \quad q_{3}=s / 2-\mu / 4+O\left(\mu^{2}\right)$,

with the equation on the integral manifold

$\mu \dot{s}=s+2 \mu s+\mu s^{2} / 4+O\left(\mu^{2}\right)$.

Figure 1 demonstrates the closeness of solutions of the original system and the system on the slow invariant manifolds for $q_{1}(t)$. The similar situation takes place for $q_{2}$ and $q_{3}$.

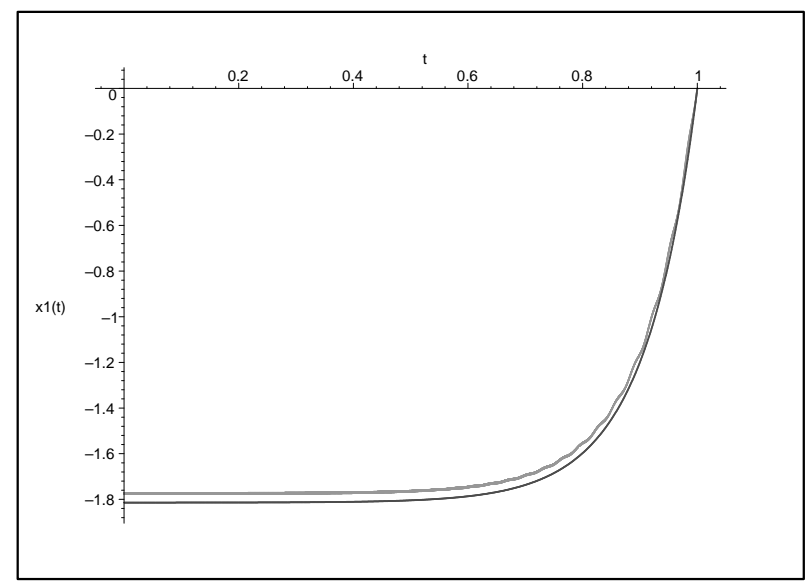

Fig. 1. The graphs of $q_{1}$ for original differential system (grey line) and for the equation on the slow invariant manifold (black line).

\section{Conclusion}

Critical cases for singularly perturbed differential systems are studied in the paper. We have considered singularly perturbed control problems as applications. It has been shown that the reduction of dimensions of these problems can be done by means of the integral manifold method. The slow integral manifolds for the matrix Riccati equation of linear-quadratic control problem are constructed and it is shown that the method of integral manifolds allows us to reduce the dimension of control problems. This approach was used for the investigation of optimal filtering problems in $[28,29]$.

\section{Acknowledgements}

This work is supported in part by the Ministry of education and science of the Russian Federation in the framework of the implementation of Program of increasing the competitiveness of SSAU for 2013-2020 years. 


\section{References}

1. Vasilieva AB, Butuzov VF, Kalachev LV. The Boundary Function Method for Singular Perturbation Problems. Philadelphia: SIAM, 1995.

2. O’Malley RE. Singular Perturbation Methods for Ordinary Differential Equations. In: Appl. Math. Sci. New-York: Springer-Verlag, 1991; 89.

3. Mortell MP, O’Malley RE, Pokrovskii A, SobolevVA. Singular Perturbation and Hysteresis. Philadelphia: SIAM, 2005.

4. Shchepakina E, Sobolev V, Mortell MP. Singular Perturbations: Introduction to System Order Reduction Methods with Applications. In: Springer Lecture Notes in Mathematics, Cham: Springer International Publishing, 2014; 2114.

5. O’Malley RE. High gain feedback systems as singular singular perturbation problems. In: Prepr. Techn. Pap., 18th Joint Automat. Control Conf., New York, 1977: 1278-1281.

6. Kokotovic PV, Khalil KH, O’Reilly J. Singular Perturbation Methods in Control: Analysis and Design. Philadelphia: SIAM, 1986.

7. Vasilieva AB, Butuzov VF. Singularly Perturbed Equations in the Critical Case. Tech. Report MRC-TSR. Madison: University of Visconsin, 1980; 2039.

8. Strygin VV, Sobolev VA. Effect of geometric and kinetic parameters and energy dissipation on orientation stability of satellites with double spin. Cosmic Research, 1976; 14: 331-335.

9. Sobolev VA. Integral manifolds and decomposition of singularly perturbed systems. System and Control Letters, 1984; 5: 169-179.

10.Gu Z, Nefedov NN, O’Malley RE. On singular singularly perturbed initial values problems. SIAM J Appl Math, 1989; 49: 1-25.

11.Kononenko LI, Sobolev VA. Asymptotic expansion of slow integral manifolds. Siberian Math J, 1994; 35: 1119-1132.

12.Sobolev VA, Strygin VV. Permissibility of changing over to precession equations of gyroscopic systems. Mechanics of Solids, 1978; 5: 7-13.

13.Shchepakina EA. Black swans and canards in self-ignition problem. Nonlinear Analysis: Real World Applications, 2003; 4: 45-50. DOI: 10.1016/S1468-1218(02)00012-3.

14.Shchepakina E. Critical conditions of selfignition in dusty media. Journal of Advances in Chemical Physics, 2001; 20(7): 3-9.

15.Shchepakina E. Slow integral manifolds with stability change in the case of a fast vector variable. Differential Equations, 2002; 38: 1146-1452.

16.Shchepakina EA. Two forms of stability change for integral manifolds. Differential Equations, 2004; 40(5): 766-769.

17.Shchepakina E. Canards and black swans in model of a 3-D autocatalator. Journal of Physics: Conference Series, 2005; 22: 194-207. DOI: http://dx.doi.org/10.1088/17426596/22/1/013.

18.Shchepakina E, Korotkova O. Condition for canard explosion in a semiconductor optical amplifier. Journal of the Optical Society of America B: Optical Physics, 2011; 28(8): 1988-1993. DOI: 10.1364/JOSAB.28.001988.

19.Shchepakina E, Korotkova O. Canard explosion in chemical and optical systems. Discrete and Continuous Dynamical Systems - Series B, 2013; 18(2): 495-512. DOI: 10.3934/dcdsb.2013.18.495.

20.Golodova ES, Shchepakina EA. Modeling of safe combustion at the maximum temperature. Mathematical Models and Computer Simulations, 2009; 1(2): 322-334. DOI: 10.1134/S207004820902015X.

21.Shchepakina EA. Critical phenomena in a model of fuel's heating in a porous medium. CEUR Workshop Proceedings, 2015; 1490: 179-189.DOI: 10.18287/1613- 0073-20151490-179-189.

22.Spong MW, Khorasani K, Kokotovic PV. An integral manifold approach to the feedback control of flexible joint robots. IEEE Journal of Robotics and Automation, 1987; 3(4): 291-300.

23.Ghorbel F, Spong MW. Integral manifold of singularly perturbed systems with application to rigid-link flexible-joint multibody system. International Journal of Non-linear Mechanics, 2000; 35: 133-155. 
24.O’Malley RE, Jameson A. 1975-77 Singular perturbations and singular arcs I, II. Trans Autom Control AC-20: 218-226 and AC-22: 328-337.

25.Murdock JA. Perturbations. Theory and Methods. John Wiley \& Sons Inc, 1991.

26.Smetannikova E, Sobolev V. Regularization of Cheap Periodic Control Problems. Autom Remote Control, 2005; 66(6): 903-916.

27.Sobolev VA. Singular perturbations in linearly quadratic optimal control problems. Autom Remote Control, 1991; 52(2): 180-189.

28. Osintsev MS, Sobolev VA. Reduction of dimensionality of optimal estimation and control problems for systems of low dissipativity solid bodies. Autom Remote Control, 2013; 74(8): 1334-1347.

29. Osintsev MS, Sobolev VA. Reduction of dimension of optimal estimation problems for dynamical systems with singular perturbations. Computational Mathematics and Mathematical Physics, 2014; 54(1): 45-58. DOI: 10.1134/S0965542514010102. 